DOI: https://doi.org/10.46296/ig.v4i8.0025

\title{
PAUTAS DE UN PROGRAMA DE MANTENIMIENTO Y SU IMPORTANCIA EN EL PROCESO AGROINDUSTRIAL
}

\section{GUIDELINES OF A MAINTENANCE PROGRAM AND ITS IMPORTANCE IN THE AGROINDUSTRY PROCESS}

\author{
Vera-Zambrano Roger Andrés ${ }^{1}$; Torres-Rodríguez Roberto ${ }^{2}$ \\ ${ }^{1}$ Programa de Mantenimiento Industrial, Instituto de Posgrado, Universidad Técnica de Manabí. \\ Portoviejo, Ecuador. rogerverzambrano7@gmail.com \\ ${ }^{2}$ Docente Departamento de Mecánica, Facultad de Ciencias Matemáticas, Físicas y Químicas, \\ Universidad Técnica de Manabí. Portoviejo, Ecuador. roberto.torres@utm.edu.ec
}

\section{Resumen}

La problemática en la agroindustria refiere al tope de la vida útil de sus maquinarias y equipos, que habiendo concluido su ciclo continúan en el proceso de producción, recibiendo únicamente tareas de corrección de fallas, en base a lo cual, el estudio es una revisión documental sobre la importancia del mantenimiento en las empresas agroindustriales. Se utilizó como metodología la revisión de 54 fuentes de información tales como artículos, libros y tesis doctorales con unos $60 \%$ de actualidad, de bases de datos indexadas para argumentar y fundamentar la importancia del mantenimiento industrial, el método deductivo que permitió poder organizar la información y consecuentemente concluir sobre la revisión efectuada. Como resultados se obtuvo el sustento de la importancia del mantenimiento industrial normado en la normativa ecuatoriana del Reglamento de seguridad y salud de los trabajadores y mejoramiento del medio ambiente de trabajo, capítulo IV, artículos 91 y 92; la importancia que tiene el mantenimiento considerando variables de organización, programación, control, fallos, capacitación, costos, productividad, seguridad e higiene industrial y contaminación ambiental en una media del 87,5\%. Se llegó a conclusiones particulares como lo vital que constituye el mantenimiento en los procesos de producción de la industria y la agroindustria, la cual, siendo una herramienta sustancial, debe aplicarse a todo tipo de activos fijos, es imprescindible dentro de la planta de producción de productos industriales y de bienes en general.

Palabras clave: agroindustria, mantenimiento, maquinaria, proceso, programación.

\begin{abstract}
The problem in the agribusiness of Manabí refers to the end of the useful life of its machinery and equipment, which, having concluded its cycle, continue in the production process, receiving only fault correction tasks, based on which, the study is a documentary review on the importance of maintenance in agro-industrial companies. The methodology used was the review of 54 sources of information such as articles, books and doctoral theses with $60 \%$ current, of indexed databases to argue and substantiate the importance of industrial maintenance, the deductive method that allowed to organize the information and consequently conclude on the review carried out. As a result, support was obtained of the importance of industrial maintenance regulated in the Ecuadorian regulations of the Regulation of health and safety of workers and improvement of the work environment, chapter IV, articles 91 and 92; the importance of maintenance considering variables of organization, programming, control, failures, training, costs, productivity, safety and industrial hygiene and environmental contamination in an average of $87.5 \%$. Particular conclusions were reached such as how vital maintenance is in the production processes of industry and agribusiness, which, being a substantial tool, must be applied to all types of fixed assets, it is essential within the production plant of industrial products and goods in general.
\end{abstract}

Keywords: agribusiness, maintenance, machinery, process, programming.

Información del manuscrito:

Fecha de recepción: 03 de mayo de 2021.

Fecha de aceptación: 05 de julio de 2021.

Fecha de publicación: 09 de julio de 2021. 


\section{Introducción}

El mantenimiento siempre ha existido, si se toma en cuenta que al proporcionar mantenimiento a una herramienta, posteriormente se le dio mantenimiento a una pieza y consecuentemente a todo el equipo - maquinaria industrial, así el mantenimiento cobra vital importancia por el gran aporte que hace a la productividad empresarial, generando la confiabilidad en los procesos productivos compuestos por maquinarias y equipos disponibles y con funcionamiento óptimo, debido al desarrollo tecnológico que implica un programa de mantenimiento (García, Cárcel, \& Mendoza, 2019).

La importancia del mantenimiento conlleva un peso en las decisiones de la planta industrial para obtener ventajas con su implementación, tales como: aseguramiento de la eficiencia de la maquinaria, incremento de la vida útil de los equipos, continuidad de los procesos, mejora de la productividad, reducción de riesgos laborales y materiales, promoción de la sostenibilidad, reducción de costos, cumplimiento de la normativa vigente, de tal forma que el mantenimiento constituye una prioridad para la empresa (Qualitymant Group, 2020).

Un programa de mantenimiento es tan importante, que de su aplicación se obtiene una mejora de la productividad, considerando tres ejes que son el costo, el plazo y la calidad. Es decir que, al garantizar el funcionamiento de la maquinaria, también se cuida el presupuesto destinado para el rubro de mantenimiento y el tiempo en que se lo realiza (IMG, 2020).

Así también los beneficios de acuerdo a (Vásquez, 2017) implican, minimizar los costos ocasionados por la falla de maquinarias y equipos que paralizan el proceso de producción, proporcionar la seguridad e higiene industrial a los operarios en sus puestos de trabajo, aprovechamiento de la materia prima, mano de obra, materiales e insumos contemplados en la carga fabril, maximizar el uso de las maquinarias y equipos y maquinaria, alargando su vida útil; disminuir los costos de producción; optimizar el inventario de repuestos disponibles en stock, sin tener que comprar demás, ni sufrir la carencia de repuestos cuando se requieran; 
seguir las normas de calidad nacionales e internacionales según el caso; ser amigable con el medio ambiente en todo el proceso de producción; mejorar el uso de los recursos materiales y económicos del área de mantenimiento; llevar un control y seguimiento de las actividades del área de mantenimiento.

Algunas funciones del mantenimiento están en prevenir, corregir y revisar equipos y maquinarias (Joaquín, 2004), lo cual implica la gestión de materiales, equipos y actividades, las técnicas de diagnóstico, análisis y mejoras, y la ejecución del mantenimiento en sus tipos, correctivo, preventivo y predictivo (Robayo, 2020). De esta manera sus objetivos están encaminados a la optimización de la disponibilidad del equipo, disminución de costos, optimación de los recursos humanos y maximización de la vida de la maquinaria (Molina, 2017).

La maquinaria empleada en la industria alimentaria varía según el producto a elaborar (López, 2015), así en la agroindustria, es sustancial llevar un adecuado control de mantenimiento de maquinarias $y$ equipos, ya que con el paso del tiempo están propensos a fallas mecánicas que ocasionan paradas repentinas en los procesos de producción, causando en algunos casos cuantiosas pérdidas económicas (Alcívar, Torres, \& Gallegos, 2020)., siendo factible con el mantenimiento conservar la seguridad, la confiabilidad, el medio ambiente y la disponibilidad de las funciones de la maquinaria y equipos (Vizcaíno, 2016).

Teniendo en cuenta que una industria es una entidad jurídica dedicada a la producción de bienes o productos finales valiosos, empleando factores como la mano de obra calificada, maquinarias y equipos, y profesionales interdisciplinarios para lograr sus objetivos de producción (Marvel et al, 2011; García, et al., 2008; Díez, et al., 2002) citado por Macías, et al. (2020); ésta ha ido creciendo aplicando nuevas tecnologías y herramientas de mejoramiento para incrementar sus procesos productivos (García, González, \& Cortés, 2009).

Siendo la actividad industrial, constante y continua, requiere disponer al máximo de los activos, su 
vida útil y funcionamiento idóneo, su conservación, la calidad de los productos y productividad de la empresa, precisa la garantía para que sus procesos productivos fluyan (Cárcel, 2011; Robbins et al, 2009; Monchy, 1990; Camelo et al, 2010; Lundvall, et al., 2007), mediante una planificación técnica que reduzca la probabilidad de imprevistos y fallos en la maquinaria industrial que dificulten lograr las metas de producción (Alavedra, y otros, 2016).

Algunas cualidades que debe tener un programa de mantenimiento comprenden una mejor forma de planear y controlar el mantenimiento a aplicar, una reducción significativa del riesgo de falla o avería (Carrillo, 2019); disminución de paros imprevistos en la maquinaria y equipos (Vásquez, 2017); el costo de la prevención es mucho menor a la corrección de fallos (Robayo, 2020). Por otra parte, la no aplicabilidad de programas de mantenimiento en la industria y agroindustria genera el incremento de la carga fabril o costos generales de fabricación que consecuentemente repercuten en un incremento de los costos totales de producción, afectando finalmente el capital de operación de la empresa, ya planificado (Macías, 2018).

Siendo así, el fondo presupuestado por la empresa agroindustrial debe incluir las tareas de reparación y mantenimiento (Cerda, Chandía, \& Faúndez, 2013), para ser utilizado en el financiamiento del mantenimiento más adecuado al proceso de producción de la agroindustria (Sulca, Espinoza, \& Becerra, 2016).

El mantenimiento en los últimos años se ha constituido en una herramienta fundamental para lograr objetivos productivos y eficientes en las empresas, así, en Ecuador, la industria ha venido aplicando e incorporando nuevas tendencias que incluyen una reducción de costos en la mantención de sus plantas industriales, maquinarias y equipos, lo cual implica una reestructuración gradual, que la lleve a ser más competitivas (Aguiaza, 2016).

Es decir que la aplicación del mantenimiento en la industria ecuatoriana está cobrando vital importancia, y considera varios enfoques en su aplicación (Moreano \& Pérez, 2020), incidiendo positivamente en el presupuesto anual de la industria, al reducir tanto el tiempo como el costo de las tareas 
de mantenimiento (Moscoso, Núnez, \& Rivas, 2019), reducción de paradas de equipos y desperdicio de insumos, propendiendo a la optimización de recursos de mantenimiento e incremento de la productividad. (Jiménez, 2017), sin embargo, no se logró encontrar publicaciones que determinen su comportamiento en la provincia de Manabí.

Dentro de la industria ecuatoriana, el sector de la agroindustria ha venido incorporado algunas técnicas predictivas al proceso de producción, tales como la termografía, análisis de vibraciones, ultrasonido y análisis de aceites (Cedeño, Areválo, \& León, 2016); ya son algunas, las empresas agroindustriales que han empezado a utilizar softwares en el control de los procesos de producción, debido a sus características innovadoras, aunque esta herramienta aún se encuentra en pleno desarrollo (Álvaro, Dini, \& Stumpo, 2016), siendo los ingenios azucareros, el tipo de empresa agroindustrial que utiliza varios tipos de mantenimiento industrial, destacándose el mantenimiento predictivo, el cual ha contribuido al incremento de la productividad de esta clase de la agroindustria (Alcívar, Torres, \& Gallegos, 2020). En Manabí, sin embargo, no se encontró información que respalde las aplicaciones del mantenimiento industrial en las agroindustrias manabitas.

No obstante, en la realidad de países como Ecuador, donde el sector agroindustrial incluye a todas las actividades relacionadas con las industrias de alimentos, bebida, tabaco, textiles, químicos, cuero, caucho, madera y papel, en que para aplicar un tipo de mantenimiento como el predictivo, involucraría además contar con maquinarias y equipos más tecnificadas, cuando éstas ya han cumplido su vida útil y siguen estando dentro del proceso de producción, en gran parte de éstas, el mantenimiento que se les proporciona responde a la corrección de fallos cuando la máquina ha paralizado su trabajo (Quevedo, 2016).

De acuerdo con estudios en esta materia, el resultado se evidencia en la línea de producción que refleja deterioros como la oxidación de engranajes, empaques y abrazaderas, desprotección de conexiones eléctricas, fugas de 
agua, entre los problemas más usuales ocasionados por la falta de mantenimiento (Ramos, 2015).

Una de las situaciones frecuentes puede observarse en la producción de queso, en máquinas giratorias como la tamizadora que separa los líquidos de los sólidos, las cuales son susceptibles de sufrir desalineación, causando una sobrecarga de los ejes provocando una pérdida de lubricación e incremento de la fuerza de rozamiento (Aguiaza, 2016). Se puede evidenciar que la mayor parte de las empresas agroindustriales escasamente aplican programas de mantenimiento, pues se remiten únicamente a tomar correctivos ante la presencia de averías y fallos en la línea de producción.

Bajo estas consideraciones, puede entenderse al mantenimiento como una debilidad en el caso específico de las empresas agroindustriales como las asentadas en la provincia de Manabí, Ecuador, estando la agroindustria manabita orientada a recuperar el valor agregado de la producción agrícola y ganadera mediante el procesamiento de lácteos como queso, yogurt $y$ mantequilla, es notorio el potencial que representa para el desarrollo sustentable de la provincia entera, el cual se ve mermado en el logro de los resultados de producción esperados, por cuanto el control de la producción difiere de actividades de mantenimiento industrial que pueden repotenciar las capacidades de toda la línea de producción, incrementando la productividad.

En base a la problemática enunciada en materia de mantenimiento en la agroindustria del Ecuador, el objetivo del presente estudio se centra en analizar las pautas e importancia que tiene el mantenimiento industrial en la agroindustria, de tal de tal manera que el programa de mantenimiento sea considerado como un componente vital que contribuye al mejoramiento de la productividad agroindustrial.

\section{Materiales y métodos}

Para poder realizar un análisis de las pautas e importancia del mantenimiento industrial en la agroindustria se realizó una revisión bibliográfica de 54 fuentes de información, extraídas de tesis de posgrado de diversas instituciones de educación superior ecuatorianas y extranjeras, libros de reconocidos 
autores y artículos científicos de apreciarse en la tabla

(1) $a$ reciente actividad, tal como puede continuación.

Tabla 1. Fuentes de información consultadas.

\begin{tabular}{|c|c|c|}
\hline Fuente de información & $\#$ & Actualidad (\%) \\
\hline Tesis & 18 & $68 \%$ \\
\hline Artículos & 17 & $65 \%$ \\
\hline Libros & 19 & $47 \%$ \\
\hline & Total 54 & Media $60 \%$ \\
\hline
\end{tabular}

Fuente: Autores (2021).

El impacto que reflejan los estudios revisados se centra no solamente en la actualidad de estos trabajos, sino en sus contenidos, así, los artículos científicos proporcionaron información reciente sobre el mantenimiento, en los libros se encontraron los principios y fundamentos que permitieron argumentar sobre la importancia del mantenimiento y la aplicación de los programas de mantención en el campo agroindustrial.

Teniendo en consideración el objetivo de la investigación, referente a las pautas e importancia del mantenimiento industrial en la agroindustria, se utilizaron fuentes primarias de información que permitieron la recolección de datos tales como como libros, revistas técnicas y científicas. Como método se empleó el razonamiento deductivo, con el cual, se partió de la lectura de varias fuentes sobre la caracterización del mantenimiento industrial estudiada por varios autores, para poder reflexionar en sus teorías y consecuentemente, concluir en la importancia del mantenimiento industrial para la industria y la agroindustria específicamente, en base a estos trabajos desarrollados, puesto que "mediante la deducción se derivan las consecuencias particulares 0 individuales de las inferencias o conclusiones generales aceptadas" (Abreu, 2014).

\section{Resultados y discusión}

Teniendo en cuenta autores como (Carrillo, 2019), (Cárdenas \& Hernández, 2018), (Castro, 2016), sustentan la importancia que presenta el mantenimiento en la industria para la preservación de la integridad física de los trabajadores, 
ya que "hay vidas que dependen del buen funcionamiento del equipo", además de la alta significancia que tiene la aplicación del mantenimiento para evitar costos innecesarios en la producción o para de la producción que provocan pérdidas sustanciales para la empresa cuando las maquinarias y equipos fallan (García S. , 2019), (Vásquez, 2017), (Molina, 2017), (García O. , 2012).

De manera que (Cárdenas \& Hernández, 2018) en sus conclusiones expresa que no imagina que existan empresas sin una política de mantenimiento, evidenciando su desinterés con respecto al mantenimiento de sus equipos en las diferentes áreas. Por último indica que nunca es tarde para comenzar si de mantenimiento se trata.

En la misma razón, la importancia del mantenimiento industrial en Ecuador se sustenta entre otros, en el Reglamento de seguridad y salud de los trabajadores y mejoramiento del medio ambiente de trabajo, el cual en el capítulo IV norma la utilización y mantenimiento de las maquinarias en la industria, considerando en el artículo 91 su utilización, así:
1. Las máquinas se utilizarán únicamente en las funciones para las que han sido diseñadas. 2 . Todo operario que utilice una máquina deberá haber sido instruido y entrenado adecuadamente en su manejo y en los riesgos inherentes a la misma. Asimismo, recibirá instrucciones concretas sobre las prendas y elementos de protección personal que esté obligado a utilizar.

3. No se utilizará una máquina si no está en perfecto estado de funcionamiento, con sus protectores y dispositivos de seguridad en posición y funcionamiento correctos.

4. Para las operaciones de alimentación, extracción y cambio de útiles, que por el peso, tamaño, forma o contenido de las piezas entrañen riesgos, se dispondrán los mecanismos y accesorios necesarios para evitarlos (Asamblea Nacional, 2012).

Así también, esta misma normativa refiere en el artículo 92 cómo debe ser el mantenimiento en la industria: 
1. El mantenimiento de máquinas deberá ser de tipo preventivo y programado.

2. Las máquinas, sus resguardos y dispositivos de seguridad serán revisados, engrasados y sometidos a todas las operaciones de mantenimiento establecidas por el fabricante, 0 que aconseje el buen funcionamiento de estas.

3. Las operaciones de engrase y limpieza se realizarán siempre con las máquinas paradas, preferiblemente con un sistema de bloqueo, siempre desconectadas de la fuerza motriz y con un cartel bien visible indicando la situación de la máquina y prohibiendo la puesta en marcha. En aquellos casos en que técnicamente las operaciones descritas no pudieren efectuarse con la maquinaria parada, serán realizadas con personal especializado y bajo dirección técnica competente (Asamblea Nacional, 2012).

Por esto (Walter Cajas, 2019) en su investigación en la implementación del mantenimiento preventivo a una empresa agroindustrial consiguió comprobar, demostrar y afirmar, que su propuesta incrementa la productividad en un $28.55 \%$ además de incrementar la eficiencia en un $13.05 \%$ e incrementar la eficacia en un $15.61 \%$, aceptando las hipótesis de su investigación.

Teniendo en cuenta que el mantenimiento correctivo es el más común, según Crespo, Moreu, \& Sánchez (2004), Muñoz (2005), Ruiz (2019) nos indica que inicia cuando aparece la falla para reparar las averías en la maquinaria y equipos, cuyo objetivo es que el equipo funcione con normalidad rápidamente para no afectar la producción.

Del mismo modo (Castillo L., 2020) en los resultados obtenidos determino que con un programa de mantenimiento espera reducir el número de falla de 87 a 64, reduciendo la perdida por los mantenimientos correctivos, dando un impacto positivo para la empresa agroindustrial la cual incrementa del $70.6 \%$ a $88.2 \%$ su eficiencia.

A razón de esto (Ruiz, 2019) resalta y concluye en los resultados de su investigación que algunas empresas en el sector agroindustrial no realizan un mantenimiento predictivo como se debe, y resalta que esto se 
debe a que las herramientas adecuadas tienen un costo elevado, dándose sobre todo en empresas la cual sus equipos no son muy costosos.

En vista de esto los autores García (2019), Rico (2018), Castro (2016),
García (2012), en los resultados de sus investigaciones, analizados de acuerdo con la característica del mantenimiento en la industria, obtuvieron la siguiente resultante evidenciada en la tabla (2) a continuación:

Tabla 2. Análisis del mantenimiento industrial.

\begin{tabular}{|c|c|}
\hline Variables de Diagnóstico & Porcentaje (\%) \\
\hline Organización & 72.6 \\
\hline Planeación - programación & 69.1 \\
\hline Control de la calidad & 70 \\
\hline Importancia & 100 \\
\hline Fallas & 90 \\
\hline Capacitación & 100 \\
\hline Costos & 100 \\
\hline Productividad & 100 \\
\hline Seguridad industrial & 100 \\
\hline Higiene industrial & 83 \\
\hline Contaminación ambiental & \\
\hline
\end{tabular}

Fuente: Revisión documental (2021).

Así también, Verdezoto (2015), Cedeño, Areválo, \& León (2016), Molina (2017), Tello (2018), García (2019), nos indican en sus revisiones documentales que el mantenimiento predictivo o basado en la condición los autores concluyen que las empresas deberían en tomar consideración este tipo de mantenimiento la cual los ayudaría en la detección anticipada de fallos y averías, evitando paros en la producción no programados, utilizando la tecnología y parámetros de control como la inspección visual, acústica y otras técnicas de monitoreo.

De modo que (Alcívar, Torres, \& Gallegos, 2020) nos da a conocer en su discusión en el área de mantenimiento en el sector agroindustrial, resalta que existe una 
escasez de mano de obra (11\%) y la falta de asistencia técnicamantenimiento (11\%) para el sector de la costa, dándole importancia a la gestión en los procesos del área de mantenimiento.

Por consiguiente, en los resultados obtenidos de (Reyes, 2021) en su simulación de un plan de mantenimiento propuesto para una empresa agroindustrial revelo que la disponibilidad aumentara un 11\% según sus resultados y los costos disminuirán un $10.77 \%$ en un mismo periodo de evaluación. Lo cual a su vez recomienda establecer los mismos indicadores para una excelente gestión del mantenimiento.

Finalmente, en los resultados obtenidos por (Revolledo, 2021) indica que los resultados obtenidos de su investigación no son tan favorables, lo cual se ven en la necesidad de proponer un plan 0 programa de mantenimiento preventivo con la finalidad de aumentar la disponibilidad y confiabilidad. Como principal objetivo se obtengan más horas de funcionamiento y reparar las fallas en menor tiempo posible.
Lo dicho, se sustenta en los resultados de la revisión bibliográfica de los hallazgos y conclusiones de los trabajos de varios autores, en materia de mantenimiento en la agroindustria, en base a lo cual, se parte de los tipos de mantenimiento que pueden aplicarse en el sector agroindustrial, así, de acuerdo a la necesidad de mantenimiento, la empresa puede asumir la que más se corresponda con su realidad operativa, el estado técnico de sus equipos e instalaciones, la cualificación de su mano de obra y del personal del área de mantenimiento, así se puede proporcionar las formas correctiva, preventiva, proactiva y predictiva.

\section{Conclusiones}

Por todo lo expuesto en los resultados y discusión se determina que el mantenimiento industrial es vital dentro de los procesos de producción de la industria y la agroindustria, la cual, siendo una herramienta sustancial, debe aplicarse a todo tipo de activos fijos, es imprescindible dentro de la planta de producción de productos industriales y de bienes en general. 
Es el mantenimiento una clave que viabiliza la generación de productos finales valiosos, promueve el aumento de la productividad de la empresa agroindustrial y coadyuva a favorecer el ambiente productivo en el galpón industrial.

La programación del mantenimiento industrial constituye una inversión a mediano o largo plazo que implica muchas ventajas para las empresas industriales, destacando el componente de la calidad en los productos que fabrica la empresa.

\section{Bibliografía}

Abreu, J. (2014). Research Method. Daena: International Journal of Good Conscience. 9(3). ISSN 1870-557X, 195-204. Retrieved from http://www.spentamexico.org/ v9-n3/A17.9(3)195-204.pdf

Aguiaza, J. (2016). DISEÑO UN PLAN DE MANTENIMIENTO PREVENTIVO PREDICTIVO PARA LA PLANTA DE PRODUCCIÓN DE LA EMPRESA ELECTRIFICACIONES DEL ECUADOR S.A "ELECDOR". Quito: ESCUELA POLITÉCNICA NACIONAL. Retrieved from https://bibdigital.epn.edu.ec

Alavedra, C., Gastelo, Y., Méndez, G., Minaya, C., Pineda, B.,
Prieto, K., . . . Moreno, C. (2016). Gestión de mantenimiento preventivo y su relación con la disponibilidad de la flota de vehículos. Ingeniería industrial, $\quad 34(1), \quad$ 11-26. Retrieved from https://www.redalyc.org/pdf/3 374/337450992001.pdf

Alcívar, M., Torres, J., \& Gallegos, F. (2020). "Gestión por Procesos en el área de Mantenimiento del Sector Agroindustrial Sucroalcoholero

Ecuatoriano". Observatorio de la Economía Latinoamericana. Retrieved from

https://www.eumed.net/rev/oe 1/2020/07/sector-

sucroalcoholero-ecuador.html

Álvaro, C., Dini, M., \& Stumpo, G. (2016). Los desafíos del Ecuador para el cambio estructural con inclusión social. Santiago: CEPAL. Retrieved from https://repositorio.cepal.org/bi tstream/handle/11362/40863/ 1/S1601309_es.pdf

Asamblea Nacional. (2012). Reglamento de seguridad y salud de Iso trabajadores y mejormaiento del medio ambiente de trabajo. Quito, Pichincha, Ecuador: Suplemento del Registro Oficial No.

Baquero, M., \& Lucio, A. (2010). La Agroindustria ecuatoriana: un sector importante que 
requiere de una ley que promueva su desarrollo. La Granja, 44-46. Retrieved from https://www.redalyc.org

Cárcel, F. (2014). La gestión del conocimiento en la ingeniería del mantenimiento industrial. Investigación sobre la incidencia en sus actividades estratégicas. Espana: OMNIA SCIENCE. Retrieved from https://books.google.es/books ?hl=es\&lr=\&id=-

Xn5AgAAQBAJ\&oi=fnd\&pg= PP1\&dq=importancia+de+los +programas+de+mantenimie nto+industrial\&ots=OCs8i8nr Vv\&sig=SK42ErD1_ZEZvSf2 ZTvMTPWfn1s\#v=onepage\& $q=$ importancia\%20de\%20los \%20programas\%20de\%20ma ntenimiento\%20industri

Cárdenas, J., \& Hernández, R. (2018). El mantenimiento industrial en Cuba y su importancia actual. Cuba: Universidad de Matanzas. Retrieved from http://monografias.umcc.cu/m onos/2018/FCE/mo18253.pdf

Carrillo, I. B. (2019). Planificación, Programación y evaluación de la Gestión de mantenimiento. Quito: Escuela politécnica nacional.

Castillo, L. (2020). Propuesta de mejora en las operaciones del área de proyecciones para incrementar la eficiencia de una empresa agroindustrial. Trujillo, Perú: Universidad Privada Del Norte. Retrieved from

https://repositorio.upn.edu.pe/ bitstream/handle/11537/2461

4/Castillo\%20L\%c3\%b3pez\% 20Liz\%20Mait\%c3\%a9.pdf?s equence $=6 \&$ is Allowed $=y$

Castro, L. (2016). Mantenimiento industrial: la columna vertebral de su empresa. Procesos, 28-32. Retrieved from

http://www.ingenieriademetod os.com

Cedeño, E., Areválo, M., \& León, D. (2016). Estudio del impacto logístico - técnico que genera el mantenimiento predictivo en las PYMES de Milagro, Ecuador. REVISTA DE CIENCIA E INVESTIGACIÓN, 7-15. Retrieved from https://dialnet.unirioja.es/servl et/articulo?codigo $=6118750$

Cerda, R., Chandía, A., \& Faúndez, M. (2013). Gestión de operaciones en empresas agropecuarias", Tópico III. Chile: Programa Gestión Agropecuaria de Fundación Chile. Retrieved from http://www.uco.es/zootecniay gestion/img/pictorex/01_16_5 4_Contabilidad_de_Gestion_ Agropecuaria.pdf

Chang, E. (2008). PROPUESTA DE UN MODELO DE GESTIÓN DE MANTENIMIENTO PREVENTIVO PARA UNA PEQUEÑA EMPRESA DEL RUBRO DE MINERÍA PARA REDUCCIÓN DE COSTOS DEL SERVICIO DE 
ALQUILER.

Lima:

UNIVERSIDAD

PERUANA

DE CIENCIAS APLICADAS.

Retrieved

from

https://repositorioacademico.

upc.edu.pe

Crespo, M., Moreu, D., \& Sánchez, H. (2004). Inegniería del mantenimiento. Técnicas y métodos de aplicación en la fase operativa de los equipos. Madrid: Ediciones AENOR.

García, G., González, H., \& Cortés, E. (2009). METODOLOGÍA DE MANTENIMIENTO CON POSIBLE APLICACIÓN EN EL SECTOR

AGROINDUSTRIAL. Revista CES Medicina Veterinaria y Zootecnia, IV(2), 137-150. Retrieved from http://www.redalyc.org.

García, J., Cárcel, J., \& Mendoza, J. (2019). Importancia del mantenimiento, aplicación a una industria textil y su evolución en eficiencia. 3C Tecnología. Glosas de innovación aplicadas a la pyme, 8(2), 50-67. Retrieved from

https://www.3ciencias.com/w p-

content/uploads/2019/06/3C-

TECNO-ED.-30_VOL.-

8_N\%C2\%BA-2_art-3-1.pdf

García, O. (2012). Gestión Moderna del Mantenimiento Industrial. Colombia: Ediciones de la U. Retrieved from https://books.google.es
García, S. (2010). La contratación del mantenimiento industrial. Madrid: Ediciones Díaz de Santos. Retrieved from https://books.google.es/books ?hl=es\&|r=\&id=LwLk1NwRXy oC\&oi=fnd\&pg=PR1\&dq=tare as+del+mantenimiento+indus trial\&ots $=\mathrm{i} A H \mathrm{HV}$ sydpl\&sig $=\mathrm{vF}$ Y6Dj55zRi6dew6FkIGYoA4IU $\mathrm{o} \# \mathrm{v}=$ onepage $\& \mathrm{q}=$ tareas $\% 20 \mathrm{~d}$ el\%20mantenimiento\%20indu strial\& $f=f a l s e$

García, S. (2012). Ingeniería de mantenimiento. Manual práctico para la gestión eficaz del mantenimiento industrial. España: Renovetec.

García, S. (2019). Ingeniería del mantenimiento. Salamanca, España: Renovetec. Retrieved from http://renovetec.com/index.ph $\mathrm{p} / 373$-ingenieria-demantenimiento

Gonzáles, F. (2010). Teoría y práctica del mantenimiento industrial avanzado, 20 edición. Madrid: Fundación CONFEMETAL. Retrieved from

https://books.google.es/books $? \mathrm{hl}=\mathrm{es} \& \mathrm{l}=\& \mathrm{id}=\mathrm{O} z \mathrm{wXOAKv}$ QAC\&oi=fnd\&pg $=P P 11 \& d q=t$ areas+del+mantenimiento+in dustrial\&ots=8Yn4EzQ8fs\&si $\mathrm{g}=\mathrm{q} 31 \mathrm{pjNbhR} 1$ -

QGfvV12VYtwLpjhc\#v=onepa ge\&q=tareas $\% 20$ del\%20mant enimiento $\% 20$ industrial $\& \mathrm{f}=\mathrm{fal}$ se 
Gonzales, R., \& García, E. (2011). Innovacion abierta; Un modelo preliminar adesde la festion del conocimiento. Intangible capital, 7(1), 82115. Retrieved from https://www.redalyc.org/pdf/5 49/54918729003.pdf

IMG. (2020). Equipos industriales: Importancia del mantenimiento industrial como mejora de la productividad. Revista IMG, 13. Retrieved from https://www.revistaimg.com/i mportancia-del-

mantenimiento-industrialcomo-mejora-de-laproductividad/

Jiménez, P. (2017). Plan de mantenimiento centrado en la confiabilidad (RCM) para el área de bunchado en la planta Electrocables. Guayaquil: Universidad de Guayaquil. Retrieved from http://repositorio.ug.edu.ec/bit stream/redug/24496/1/TESIS \%20JIMENEZ\%20ZAMBRAN O\%20PABLO.pdf

Joaquin, A. (2004). TECNICAS DE MANTENIMIENTO INDUSTRIAL. Retrieved from Academia.edu: https://www.academia.edu

López, M. (2015). El mercado de la maquinaria agroindustrial en Marruecos. Marruecos: ICEX, España Exportación e Inversiones. Retrieved from https://www.camarazaragoza. com
Macías, et al. (2020). Industrial engineering in higher education processes in zone 4: Ecuador. International Journal of Advanced Science and Technology, 29(8), 5377 5388.

Macías, T. (2018). Análisis comparativo de la producción orgánica y convencional de Theobroma cacao en el cantón Bolívar. Portoviejo: Universidad Técnica de Manabí.

Mayorga, A., \& Quishpe, J. (2019). Deontología aplicada al mantenimiento de maquinaria industrial por ingenieros mecánicos. Revista Caribeña de Ciencias Sociales, 1-8. Retrieved from https://www.eumed.net/rev/ca ribe/2019/05/deontologiamaquinaria-industrial.htm

Medrano, J., Gónzales, V., \& Díaz, V. (2017). Mantenimiento: técnicas y aplicaciones industriales. Caalunya. Retrieved from https://bok.lat/book/5867357/1adaed? id $=5867357 \&$ secret $=1$ adaed

Molina, J. (2017). MANTENIMIENTO Y SEGURIDAD INDUSTRIAL. Macaray: UNIVERSIDAD NACIONAL DE SAN LUIS. Retrieved Noviembre 18, 2020, from Mantenimiento $y$ seguridad industrial: www.ugr.unsi.edu.ar

Moreano, F., \& Pérez, E. (2020). Plan de mantenimiento 
preventivo para la mejora del índice de falla de un sistema de transporte neumático. Dominio de las ciencias, 6(4). Retrieved from https://dominiodelasciencias.c om/ojs/index.php/es/article/vi ew/1469/html

Moscoso, R., Núñez, S., \& Rivas, E. (2019). Programa de control de mantenimiento proactivo de equipos mecánicos utilizados en el transporte de hidrocarburos en Ecuador. Revista mktDescubre ESPOCH FADE, 111-118. Retrieved from http://revistas.espoch.edu.ec

Munoz, R., Macías, T., Andrade, F., \& Hernández, A. (2020). Design and construction of an industrial ship conditioning system. International Journal of Physical Sciences and Engineering, 4(1), 29-38. doi: https://doi.org/10.29332/ijpse. v4n1.423

Muñoz,

B.

(2005)

MANTENIMIENTO

INDUSTRIAL.

Madrid:

Universidad Carlos III de Madrid. Retrieved from http://ocw.uc3m.es

Olarte, W., Botero, M., \& Cañón, B. (2010). Importancia del mantenimiento industrial dentro de los procesos de producción. Scientia et Technica, 44(1), 354-58. Retrieved from ww.dialnet.unirioja.es
Organización para la Cooperación y el Desarrollo Económico. (2019). Generalidades del mantenimiento. Retrieved from oecd.org: https://www.oecd.org/centrod emexico/laocde/

Ortiz, A., Rodríguez, C., \& Izquierdo, H. (2013). Gestión de mantenimiento en pymes industriales. Revista Venezolana de Gerencia, 18 (61), 86-104. Retrieved from https://www.redalyc.org/pdf/2 90/29026161004.pdf

Qualitymant Group. (2020, abril). Qualitymant.com. Retrieved from La importancia del mantenimiento industrial: https://qualitymant.com/laimportancia-delmantenimiento-industrial/

Quevedo, T. (2016). Agroindustria y concentración de la propiedad de la tierra. Quito: OCARU. Retrieved from http://biblioteca.clacso.edu.ar/ Ecuador/iee/2017062703580 8/pdf_471.pdf

Ramos, J. (2015). Diagnóstico y diseño de un plan de mantenimiento preventivo para la maquinaria y equipo agroindustrial. Honduras: Zamorano. Retrieved from https://bdigital.zamorano.edu/ bitstream/11036/1551/1/AGI2002-T034.pdf

Revolledo, J. (2021, 06). Plan de Mantenimiento preventivo para la maquinaria Agrícola, 
en la Empresa Agronegocios Arteaga S.A.C, Jaén. 2021. Jaén, Perú. Retrieved from http://repositorio.unj.edu.pe/bi tstream/handle/UNJ/379/Rev olledo_VJD.pdf?sequence $=1$ \&isAllowed $=\mathrm{y}$

Reyes, V. (2021). Disminución de los costos de operación en las cosechadoras de caña a través de un plan de mantenimiento en la empresa agroindustrial laredo S.A.A. Trujillo, Perú. Retrieved from https://repositorio.ucv.edu.pe/ bitstream/handle/20.500.1269 2/60446/Ventura_RAG-

SD.pdf?sequence $=1$ \&isAllow ed $=y$

Rico, J. (2018). ESTRUCTURACIÓN DE UN MODELO DE GESTIÓN DEL MANTENIMIENTO PREVENTIVO APLICABLE A LA INDUSTRIA DE MOLINERÍA DE ARROZ EN EL DPTO. DEL TOLIMA. Ibagué: UNIVERSIDAD DE IBAGUÉ. Retrieved from https://repositorio.unibague.e du.co

Robayo, N. (2020). Diseño y programación de un plan de mantenimiento preventivo para los equipos e instalaciones de una institución de educación superior. Quito: Escuela Politécnica Nacional.

Ruiz, Á. (2019). CARACTERIZACIÓN DE LA FUNCIÓN MANTENIMIENTO
EN EL SECTOR AGROINDUSTRIAL DE LA REGIÓN PIURA. Piura: UNIVERSIDAD DE PIURA. Retrieved from https://pirhua.udep.edu.pe

Sierra, G. (2004). PROGRAMA DE MANTENIMIENTO

PREVENTIVO PARA LA EMPRESA

METALMECANICA INDUSTRIAS AVM S.A. Bucaramanga:

UNIVERSIDAD INDUSTRIAL DE SANTANDER. Retrieved from

http://tangara.uis.edu.com

Sulca, G., Espinoza, V., \& Becerra, E. (2016). Contabilidad agrícola. Quito: Cámara Ecuatoriana del libro, Universidad de las Fuerza Armadas (ESPE). Retrieved from

http://www.dspace.uce.edu.e c/bitstream/25000/21009/1/C ontabilidad\%20agr\%C3\%ADc ola.pdf

Tello, A. (2018). GESTIÓN ADMINISTRATIVA $Y$ OPERATIVIDAD DE LOS EQUIPOS INDUSTRIALES EN LOS TALLERES LABORATORIOS DE MECANIZADO DE CIYA. INVPOS, 1-11. Retrieved from http://investigacion.utc.edu.ec /revistasutc/index.php/invpos/ article/view/197/169

Vásquez, C. (2017). Gestión y planificación del mantenimiento industrial. 
Lima, Perú: Integra markets.

Retrieved from

https://www.academia.edu/36 808003/Gestion_y_Planificaci on_del_Mantenimiento_Indus trial

Verdezoto, N. (2015). PROPUESTA DE ELABORACION DE UN PLAN DE MANTENIMIENTO PREDICTIVO, BASADO EN LA CRITICIDAD DE LOS EQUIPOS DEL PROCESO DE LAMINACION EN CALIENTE PARA LA EMPRESA ANDEC S.A.". Guayaquil: Universidad de Guayaquil.

Vizcaíno, M. (2016). Desarrollo de un plan modelo de mantenimiento para el funcionamiento adecuado de los equipos eléctricos y mecánicos. Riobamba: Escuela Superior Politécnica del Chimborazo. Retrieved from

http://dspace.espoch.edu.ec/ bitstream/123456789/4752/1/ 20T00718.pdf

Walter Cajas, A. D. (2019). Implementación de un plan de mantenimiento preventivo para mejorar la productividad en la empresa Agroindustria Santa María SAC, Independencia, 2019. Lima, Perú. Retrieved from https://repositorio.ucv.edu.pe/ bitstream/handle/20.500.1269 2/50526/Cajas_AWSDel\%20\%c3\%81guila_P\%c3 $\% 81$ DJ-
SD.pdf?sequence=1\&isAllow

ed $=y$ 